\title{
Validação do Questionário de Avaliação de Habilidades Sociais, Comportamentos, Contextos para Universitários
}

\author{
Alessandra Turini Bolsoni-Silva ${ }^{1}$ \\ Sonia Regina Loureiro \\ Universidade Estadual de São Paulo
}

\begin{abstract}
RESUMO - Verifica-se uma carência de instrumentos que avaliem habilidades sociais na interface com a saúde mental de universitários. Objetiva-se testar as propriedades psicométricas de validade e fidedignidade do Questionário de Avaliação de Habilidades Sociais, Comportamentos e Contextos para Universitários - QHC-Universitários. Procedeu-se à avaliação, em situação coletiva, de 609 estudantes universitários de diferentes cursos por meio dos seguintes instrumentos: QHC-Universitários, IHS-Del Prette, BDI, Mini-SPIN e SCID-IV. As propriedades psicométricas foram aferidas por meio de procedimentos estatísticos, com resultados satisfatórios quanto à consistência interna e às validades de construto e concorrente. A análise fatorial isolou três fatores para a Parte 1 (Comunicação e Afeto, Enfrentamento, Falar em Público) e dois fatores para a Parte 2 (Potencialidades, Dificuldades), fornecendo escores de problemas e de habilidades.
\end{abstract}

Palavras-chave: habilidades sociais, saúde mental, estudantes universitários, medidas

\section{Validation of the Social Skills, Behaviors and Context Assessment Questionnaire for University Students}

\begin{abstract}
There is a lack of instruments to assess social skills in the interface with mental health of college students. This study intended to test the psychometric properties of validity and reliability of the Social Skills, Behaviors and Context Assessment Questionnaire for University Students (QHC - University Students). In a collective situation 609 university students from different courses were evaluated with the following instruments: QHC - College students, IHS-Del Prette, BDI, Mini-SPIN and SCID-IV. The psychometric properties were measured by statistical procedures, with satisfactory results concerning the internal consistency and construct validity and concurrent. Factor analysis isolated three factors for Part 1 (Communication and Affect, Coping, Public Speaking) and two factors for Part 2 (Potentialities and Difficulties), providing scores of problems and skills.
\end{abstract}

Keywords: social skills, mental health, college students, measurement

A prevalência de problemas de saúde mental em estudantes universitários é alta, o que sugere necessidade de identificação, prevenção e tratamento precoce (World Health Organization, 2004), especialmente por tratar-se de um período de vida decisivo para as atividades da vida adulta. Ramírez, Hernández e García-Campayo (2009) identificaram que $25,4 \%$ dos estudantes apresentavam algum grau de depressão e $50 \%$, de ansiedade.

Os problemas de saúde mental de universitários foram estudados por diferentes autores. Fang et al. (2010) constaram que, no início do curso, os estudantes apresentavam maiores índices de depressão. Com base em estudo conduzido com 1027 estudantes de nove faculdades de medicina públicas ou privadas de diferentes regiões dos Estados Unidos, Roberts et al. (2001) identificaram que 19\% referiram manifestações de ansiedade $18 \%$ de depressão, e $46 \%$ relataram ter tido pelo menos um sintoma de problema relativo à saúde mental. No Brasil, Neves e Dalgalarrondo (2007) identificaram que $85 \%$ dos universitários investigados relataram ao menos um transtorno mental.

1 Endereço para correspondência: Avenida Eng ${ }^{\circ}$ Luiz Edmundo Carrijo Coube, $\mathrm{n}^{\circ}$ 14-01, Vargem Limpa, Bauru, SP, Brasil. CEP:17033-360. E-mail: bolsoni@fc.unesp.br
A saúde mental do universitário tem sido associada a uma diversidade de variáveis, tais como (a) morar sozinho ou com os familiares (Cerchiari, Caetano, \& Faccenda, 2005; Facundes \& Ludermir, 2005); (b) a natureza da atividade prevista no curso (Adlaf, Gliksman,.., Demers \& NewtonTaylor, ., 2001; Facundes \& Ludermir, 2005; Neves \& Dalgalarrondo, 2007); (c) o momento do curso (Cerchiari et al, 2005); (d) o sexo (Barreto et al., 2004); (e) repertório de habilidades sociais (Veenman, Wilhelm \& Beishuizen, 2004). Tem sido destacado também que a adaptação à vida universitária pode ser associada à história prévia de vida (Facundes \& Ludermir, 2005) e às condições contextuais da vida universitária, tais como estudar diferentemente da forma como se preparou para o vestibular; lidar com autoridade (professor); falar em público (por exemplo, apresentar seminários); fazer novas amizades; morar com outras pessoas, por vezes, desconhecidas (repúblicas); negociar divisão de tarefas (repúblicas); cuidar de si mesmo e dos próprios pertences; ficar longe da família, amigos e namorado(a); administrar renda; e trabalhar para se sustentar (Ribeiro \& Bolsoni-Silva, 2011).

Configura-se, assim, um conjunto de condições que podem concorrer para o sucesso nas vivências acadêmicas e a boa qualidade de vida ou que podem favorecer experiências 
estressantes, influenciando a presença de indicadores de transtornos depressivos, de baixo rendimento acadêmico (Backer, 2003; Ciarrochi, Deane \& Anderson, 2002; Cole, Lazarick, \& Howard, 1986; Furtado et al., 2003) e inclusive de abandono escolar (Mcgaha \& Fitzpatrick, 2005).

As instituições de ensino universitário parecem ter pouca clareza dos grandes desafios e dos conflitos enfrentados pelos estudantes quanto às dificuldades relacionadas às exigências com os relacionamentos interpessoais e com as atividades acadêmicas (Gerk \& Cunha, 2006). Nesse contexto, inserese a necessidade de estudos que incluam, entre as variáveis pesquisadas, os indicadores de saúde mental e de recursos que possam favorecer a adaptação, tais como as habilidades sociais, fornecendo instrumentos aferidos para a prevenção e intervenção com essa população.

No Brasil, foram identificados três instrumentos aferidos que mensuram o repertório de habilidades sociais de universitários a partir do autorrelato, seja indiretamente (a Escala de Avaliação de Vida Acadêmica - Vendramini et al., 2004) ou diretamente (a Escala de Assertividade Rathus - Ayres, 1994; o Inventário de Habilidades Sociais - IHSDel Prette - Del Prette \& Del Prette, 2001). Analisando-se as peculiaridades desses instrumentos verifica-se que tanto a Escala de Assertividade Rathus como o IHS-Del Prette avaliam o constructo habilidades sociais, mas não têm por objetivo avaliar variáveis antecedentes e consequentes com diferentes interlocutores e sua relação com a saúde mental de universitários. A Escala de Avaliação de Vida Acadêmica aborda a vida na universidade, mas não tem como foco o repertório de habilidades sociais.

É nessa lacuna que se insere a proposição do presente estudo, desenvolvido com estudantes universitários, propondo-se a aferir um instrumento que avalie as habilidades sociais nas interações frente a diferentes interlocutores e variáveis contextuais, comparando grupos clínicos e não clínicos quanto a características de saúde mental. Objetivase testar as propriedades psicométricas de validade e de fidedignidade do Questionário de Avaliação de Habilidades Sociais, Comportamentos e Contextos para Universitários QHC-Universitários.

\section{Método}

\section{Aspectos Éticos}

O instrumento proposto é parte de um projeto mais amplo que prevê a avaliação e a intervenção junto a universitários, cujo título é "Análise das Habilidades Sociais de Grupos Universitários". O projeto foi aprovado em 30 de agosto de 2007 pelo Comitê de Ética em Pesquisa da UNESP (processo $\mathrm{n}^{\mathrm{o}}$ 1315/46/01/07). A coleta de dados foi iniciada em 2008, após a aprovação do referido Comitê de Ética.

\section{Participantes}

Participaram, na primeira etapa desse estudo, 609 estudantes universitários, graduandos de diferentes cursos de uma universidade pública do centro-oeste paulista, distribuídos em todos os anos dos cursos que frequentavam. Desses, 413 participantes (233 homens e 180 mulheres) foram considerados não clínicos com base na avaliação sistemática por meio de instrumentos que mensuram indicadores de saúde mental (vide Instrumentos) e 196 foram considerados clínicos, ou seja, apresentaram indicadores de problemas, em um ou mais desses instrumentos (83 homens e 113 mulheres). Para os estudos de fidedignidade e de validade de critério, adotaram-se dois procedimentos: no de validade convergente, foi utilizada a amostra de 609 universitários; e nos de validade concorrente foram incluídos 64 e 288 participantes, respectivamente para os Transtornos de Depressão e de Ansiedade, sendo aqueles que preencheram indicadores diagnósticos.

A caracterização dos dados sociodemográficos, acadêmicos e de relacionamentos é apresentada a seguir.

(a) Ano do curso: 179 alunos do primeiro ano, 135 do segundo, 134 do terceiro, 118 do quarto ano, 36 do quinto ano, 6 estavam fora do perfil $\left(6^{\circ}\right.$. ou $7^{\circ}$. anos $)$ e 1 não informou.

(b) Sexo: 316 homens, 293 mulheres e 3 não informaram.

(c) Ocupação: 233 trabalhavam, 373 não trabalhavam e 3 não informaram.

(d) Período do curso: 260 estudavam no período integral; 346, no noturno; e um não respondeu.

(e) Estado civil: 585 pessoas eram solteiras; 14, casadas; 4 possuíam outro estado civil e 6 não informaram.

(f) Relacionamento afetivo: 287 namoravam, 311 não namoravam e 11 não responderam.

(g) Moradia: 56 moravam sozinhos; 265, em repúblicas; 256, com a família; 14, com namorados; 13 relataram morar com outras pessoas sem identificá-las; e 5 não informaram.

(h) Cursos/áreas que frequentavam: Humanas - Arquitetura e Urbanismo $=31$, Artes Visuais $=15$, Pedagogia $=59$, Relações Públicas $=35$; Exatas - Ciências da Computação $=31$, Design $=18$, Educação Física $=$ 66, Engenharia Civil $=40$, Engenharia Elétrica $=21$, Engenharia Mecânica $=54$, Engenharia da Produção $=15$, Física $=34$, Jornalismo $=42$, Matemática $=36$, Química $=36$, Radialismo $=15$, Sistemas de Informação $=31$; Biológicas - Biologia $=29$ e 1 não informou.

(i) Idade- variou de 17 a 44 anos (média $=21,23$, desvio padrão $=3,18)$.

\section{Instrumentos}

Questionário de Avaliação de Comportamentos e Contextos para Universitários - QHC-Universitários (Bolsoni-Silva, 2011). É um questionário que avalia habilidades sociais, composto por questões que se referem à forma como o participante se comporta em relação a diversos interlocutores e contextos. A primeira parte é composta por 30 itens e conta com três fatores: 1 - Comunicação e Afeto, 2 - Enfrentamento, 3 - Falar em Público. A segunda parte organiza os 448 itens em dois fatores: Potencialidades e Dificuldades. O instrumento QHC-Universitários, dadas as suas características psicométricas aferidas foi aprovado para 
aplicação como teste psicológico pelo Conselho Federal de Psicologia em 25/10/2013 (link: http://satepsi.cfp.org.br/ listaTeste.cfm?status=1).

O QHC-Universitários foi elaborado considerando a literatura que descreve (a) déficits de habilidades sociais de universitários que buscaram por atendimento psicológico (Ribeiro \& Bolsoni-Silva, 2011); (b) relações entre habilidades sociais e saúde mental na universidade (Woods, Reed, \& Collins, 2003); e (c) a influência dos cursos de graduação na promoção de habilidades sociais (Del Prette \& Del Prette, 1983). Teve-se como norteador, na elaboração do instrumento, a avaliação de habilidades sociais e contextos, considerando as proposições de Goldiamond (1974/2002) e Sturmey (1996).

Na sua versão inicial, havia 40 itens para a Parte 1 do instrumento e 764 para a Parte 2, considerando a diversidade de possibilidades de respostas previstas pela literatura já mencionada. De forma a reduzir o número de itens, foram conduzidas análises discriminativas para as duas partes do instrumento, considerando os grupos com indicadores diagnósticos e sem indicadores diagnósticos a partir da MiniSPIN, do BDI e da entrevista clínica SCID-IV (instrumentos posteriormente descritos). Consequentemente, o QHCUniversitários, na sua versão final, apresentou apenas os itens que discriminaram os grupos, dando a ele um caráter de rastreador em saúde mental, sobretudo no que se refere à ansiedade e à depressão.

Apesar de mais reduzido, o instrumento ainda é extenso para a condução de análises de fatores a partir dos itens, então, decidiu-se por somar os itens de frequência em categorias definidas a partir de construtos teóricos (Parte 1): Comunicação (mãe, pai, irmão, amigos, namorado), sentimentos positivos (mãe, pai, irmão, amigos), sentimentos negativos (mãe, irmão, amigos, colegas, namorado), opiniões (mãe, pai, irmão, amigos, namorado), fazer críticas (colegas, namorado), receber críticas (mãe, irmão, amigos, colegas, namorado), falar em público (conhecido, desconhecido e apresentar seminários). Os itens de conteúdo (Parte 2) também foram somados em categorias, considerando construtos teóricos: situações/assuntos, comportamento habilidoso, comportamento não habilidoso, consequência positiva, consequência negativa, sentimentos positivos e sentimentos negativos, para os oito conjuntos de informação (comunicação, sentimento positivo, sentimento negativo, expressão de opinião, fazer crítica, receber crítica, falar em público e apresentar seminários).

Inventário de Habilidades Sociais- IHS-Del-Prette (Del Prette \& Del Prette, 2001). Utilizado para a avaliação da validade concorrente. É um instrumento composto por 38 itens, que tem como objetivo avaliar dimensões situacionais e comportamentais das habilidades sociais de autorrelato. Esses itens são agrupados em cinco fatores amplos: autoexposição a desconhecidos, situações novas e autocontrole da agressividade, enfrentamento e autoafirmação com risco, conversação e desenvoltura social, autoafirmação na expressão de sentimento positivo. Del Prette, Del Prette e Barreto (1998) encontraram alfa de Cronbach de 0,75 e cinco fatores com alfas variando de 0,74 a 0,96 , explicando $92,75 \%$ da variância. O IHS - Del Prette também tem boas qualidades psicométricas de validade concomitante com a escala Rathus $(r=0,81, p=0,01)$ e estabilidade temporal $(r=0,81, p=$ 0,01) (Bandeira, Costa, Del Prette \& Del Prette,., 2000).

Inventário de Depressão de Beck (BDI). Incluído no estudo como uma medida de saúde mental, enquanto um rastreador de indicadores de depressão. O instrumento foi traduzido, adaptado e validado para a população brasileira por Cunha (2001), é auto-administrado, composto por 21 itens que avaliam a gravidade dos sintomas de depressão, os quais são pontuados em uma escala likert de 0 (absolutamente não) a 3 (gravemente). Permite classificações da depressão nos níveis mínimo (0-11), leve (12-19), moderado (20-35) e grave (36-63). Originalmente criado para uso com pacientes psiquiátricos, esse instrumento também se mostrou adequado para o uso na população geral. Finger (2008) realizou um estudo com o objetivo geral de validar o referido inventário para uma população universitária brasileira, tendo relatado características psicométricas satisfatórias: Alfa de Cronbach $(0,88)$ e estabilidade temporal de 0,731 .

Versão reduzida do Inventário de Fobia Social Mini SPIN (Osório, Crippa, \& Loureiro, 2007). Avalia indicadores de fobia social, incluído como uma medida de ansiedade. Proposto por Connor et al. (2001), inclui três itens que se mostraram como os mais discriminativos de pessoas com transtorno de ansiedade social. No Brasil o instrumento foi traduzido e adaptado por Osório (2008) e, em estudo psicométrico, foi identificado Kappa igual 0,80 e coeficiente $0.84(p<0,001)$ entre aplicação face-a-face e por telefone (Osório et al., 2007).

Entrevista clínica estruturada para o DSM-IV (SCID-I - versão clínica - Del-Ben et al., 2001). Incluída no estudo como uma medida de saúde mental que permite a classificação diagnóstica por transtornos, assim como a classificação de ausência de transtorno psiquiátrico. Tratase de instrumento diagnóstico, considerado padrão ouro em estudos clínicos, utilizado para a elaboração de diagnósticos clínicos psiquiátricos baseados no DSM-IV. A entrevista foi proposta por First et al. (1997) e traduzida e adaptada para o português por Del-Ben et al. (2001). É composta por módulos, em um total de dez, que podem ser aplicados de forma independente ou combinada, conforme os objetivos da avaliação. A aplicação da SCID inicia-se por uma seção de revisão geral, que segue o roteiro de uma entrevista clínica não estruturada. Em seguida, é dividida em módulos que investigam as categorias diagnósticas maiores. Segundo Del-Ben et al. (2001), a SCID teve o Kappa ponderado excelente $(\mathrm{Kw}=0,83)$ e a fidedignidade foi estatisticamente significativa para diversos transtornos: transtorno do humor $(\mathrm{K}=0,87)$, transtornos psicóticos $(\mathrm{K}=0,90)$, transtornos relacionados ao uso de substância $(\mathrm{K}=0,76)$, transtornos de ansiedade $(K=0,61)$.

\section{Procedimentos de Coleta de Dados}

A coleta dos dados teve início após autorização das coordenações de cursos e dos respectivos professores, que foram contatados em salas de aula. Após a anuência dos mesmos, foram oferecidos aos estudantes os devidos esclarecimentos sobre os objetivos do estudo e os aspectos éticos relativos a participação. Os participantes que 
voluntariamente concordaram participar da pesquisa assinaram a um Termo de Consentimento Livre e Esclarecido e receberam uma carta explicativa sobre os objetivos do projeto e um caderno contendo instruções sobre a aplicação dos instrumentos acima descritos, com exceção da SCID, que foi conduzida por telefone posteriormente. Após a redução dos itens do instrumento, o mesmo foi reaplicado com 59 universitários, sendo que os resultados psicométricos foram, em parte, mantidos.

\section{Procedimentos de Tratamento e Análise de Dados}

Para a construção do instrumento, foram conduzidas análises discriminativas de forma a reduzir seus itens (Teste $t$ de Student), considerando para tal as classificações clínico e não clínico, a partir dos indicadores obtidos pela SCID e de depressão e ansiedade obtidos respectivamente nos instrumentos BDI e Mini-Spin. Em todas as análises, considerou-se o nível de significância de $p \leq 0,05$.

\section{Fidedignidade}

Foram conduzidas análises Alfa de Cronbach para as categorias identificadas no instrumento, nas Partes 1 e 2.

\section{Validade de Construto}

A análise fatorial foi conduzida visando verificar a validade de construto. Considerando as categorias amplas das Partes 1 e 2 do instrumento, adotou-se a análise dos componentes principais e a rotação Varimax. Após a redução dos itens, o instrumento foi reaplicado a 59 universitários, cujas análises foram repetidas de forma a verificar se os resultados se mantinham.

\section{Validade de Critério Concorrente}

Para compor os grupos de análises da validade concorrente - nos quais a administração do teste e a avaliação diagnóstica foram simultâneas -, os participantes foram distribuídos em dois grupos clínicos: Depressão e Ansiedade. Para compor o Grupo Depressão, foram incluídos os participantes com indicadores de depressão (BDI) com ou sem confirmação diagnóstica pela SCID-IV, com ou sem outras comorbidades. Para compor o Grupo Ansiedade, foram incluídos os participantes com indicadores de ansiedade (Mini SPIN) com ou sem confirmação diagnóstica pela SCID-IV. Os grupos clínicos foram comparados com um grupo não clínico. Foram utilizados o BDI, a Mini SPIN e a SCID-IV como padrões ouro para as análises de Curva Roc, de forma a identificar pontos de corte para clínico e não clínico para depressão e ansiedade.

Para a inclusão no grupo não clínico, foram alocados os participantes que não apresentaram indicadores de problema nos instrumentos de saúde mental utilizados, a saber BDI, Mini-Spin e SCID-IV.Para a composição desse grupo, o critério foi o de escolher o mesmo número de homens e de mulheres, bem como de estudantes de áreas de cursos (humanas, exatas e biológicas), previamente obtidos no grupo clínico. Dessa forma, foi possível controlar a interferência de tais variáveis. Quando se contava com mais participantes não clínicos que preenchiam os dois critérios de inclusão, esses foram sorteados.
Grupo Depressão. O Grupo de Depressão foi composto por 64 participantes (24 homens e 40 mulheres). Desses universitários, 35 responderam tanto o BDI quanto a SCID e 29 responderam apenas ao BDI. Dos universitários que responderam aos dois instrumentos, 11 pontuaram apenas na SCID depressão ${ }^{1}$ e apenas dois deles, em ambos os instrumentos (SCID e BDI). Dos 64 participantes, 31 deles também pontuaram na Mini SPIN (indicador de fobia social). Quanto à distribuição por cursos, 24 eram universitários da área de exatas; 37, da área de humanas; e 3, de biológicas. Do grupo não clínico, composto por 415 universitários, foram selecionados 64 participantes, pareados com as características do grupo clínico quanto ao sexo e área do curso. Nota-se que foi possível parear 61 participantes não clínicos, considerando ambas as variáveis (sexo e curso), os demais três participantes foram pareados apenas quanto a sexo, de forma que a amostra ficou constituída por 24 homens clínicos e 24 homens não clínicos e por 40 mulheres clínicas e 40 não clínicas.

Grupo Ansiedade. Foi composto por 144 participantes, sendo clínico para Transtornos de Ansiedade, enquanto manifestações de ansiedade com ou sem confirmação diagnóstica, mas sem comorbidades $(n=65$ pontuaram só na Mini-Spin, 55 pontuaram apenas para a SCID - Ansiedade e 24 pontuaram em ambos os instrumentos). O grupo clínico foi composto por 71 universitários da área de exatas, por 63 da área de humanas e por 9 de biológicas, 1 universitário não informou o curso. Quanto ao sexo, eram 64 homens e 80 mulheres.

Do grupo não clínico, composto por 415 universitários, foram selecionados, conforme critérios citados anteriormente, os 144 pareados com as características do grupo clínico quanto ao sexo e tipo de curso. Foi possível parear 137 participantes não clínicos considerando ambas as variáveis (sexo e curso), os demais sete participantes foram pareados apenas quanto a sexo, de forma que a amostra ficou constituída por 64 homens clínicos e 64 homens não clínicos e por 80 mulheres clínicas e 80 não clínicas.

\section{Validade Convergente}

$\mathrm{O}$ estudo da validade convergente foi realizada por meio da correlação de Pearson com o IHS-Del Prette, que mede o mesmo constructo: habilidades sociais.

\section{Resultados}

Os resultados estão organizados em tópicos: estudos de fidedignidade e estudos de validade baseados na estrutura interna com a Análise Fatorial, para as Partes 1 e 2 do QHC-Universitários. Em seguida, são apresentados os dados do estudo de validade concorrente para os indicadores de depressão e ansiedade, sendo apresentadas as Curvas ROC obtidas para cada fator das duas partes do QHCUniversitários. Na sequência, são apresentados os dados

1 Optou-se por manter na amostra apenas os 13 participantes que pontuaram na SCID em depressão, sem comorbidades. Outros três participantes pontuaram na SCID depressão e em outros transtornos, os quais foram excluídos para aumentar o controle quanto à comorbidades 
relativos à validade convergente, tendo o IHS-Del Prette como referência para as medidas de correlação.

\section{Estudo de Fidedignidade}

Os estudos de consistência interna por meio do coeficiente Alfa de Cronbach foram conduzidos para as Partes 1 e 2 do instrumento. Quanto à primeira parte, para os 30 itens que compõem o instrumento, o alfa foi de 0,657 para as oito categorias. A Parte 2 do instrumento teve também por alfa o valor de 0,657 quando agregados em sete categorias. No estudo de reaplicação, para a Parte 1, o alfa por categorias foi de 0,630 e, para a Parte 2, foi de 0,621.

\section{Estudos de Validade Baseados na Estrutura Interna - Análise Fatorial}

Para a Parte 1 do QHC-Universitários, o índice KayserMeyer-Olkin (KMO) obtido foi de 0,714 e o teste de esfericidade de Barlett apresentou resultado significativo $(p=0,000)$. Os resultados do KMO e Barlett mostraram adequação dos dados para as análises.

Conforme a Tabela 1, nota-se que o QHC-Universitários - Parte 1 apresentou três fatores que agregaram as categorias elaboradas, conforme descrito na seção de instrumentos: (a) Fator 1, denominado de Comunicação e Afeto, correspondendo a $27,3 \%$ da variância e agregando as categorias de Conversação, Expressão de Sentimentos Positivos e Expressão de Opiniões; (b) Fator 2, denominado de Enfrentamento, explica 22,2\% da variância e corresponde às categorias Expressão de Sentimentos Negativos, Faz Críticas e Recebe Críticas; (c) Fator 3, denominado de Falar em Público, explica 16,8\% da variância, correspondendo às categorias Fala em Pública e Apresenta Seminários. $\mathrm{O}$ instrumento explicou $66,3 \%$ da variância dos dados. Na tabela 1, estão descritas todas as cargas fatoriais.

Quanto à parte 2 do instrumento, o índice KMO obtido foi de 0,776 e o teste de esfericidade de Barlett apresentou resultado significativo $(p=0,000)$. Os resultados do KMO e Barlett mostraram adequação dos dados para as análises. Quanto ao QHC-Universitários - Parte 2, as categorias comportamentais foram explicadas por dois fatores. $\mathrm{O}$ primeiro, denominado Potencialidades, considerando a Rotação Varimax, agregou as categorias Situações/assuntos, Comportamento habilidoso, Consequência positiva e Sentimentos positivos. O segundo fator, Dificuldades, agregou Comportamento não habilidoso, Consequência negativa e Sentimentos negativos. A Parte 2 do QHCUniversitários explicou $71,9 \%$ da variância dos dados.

$\mathrm{Na}$ reaplicação do instrumento na sua versão final, com 49 participantes, o índice KMO obtido foi de 0,614 e o teste de esfericidade de Barlett apresentou resultado significativo $(p=0,003)$. Os resultados do KMO e Barlett mostraram adequação dos dados para as análises. As categorias da Parte 1 do QHC-Universitários também se organizaram em três fatores, no entanto, de maneira diferente da validação exploratória da versão original: (a) Fator 1 correspondendo a $29,738 \%$ da variância e agregando as categorias de Expressão de Sentimentos Positivos e Falar em Público; (b) Fator 2 explica 19,389\% da variância e corresponde às categorias Comunicação, Opiniões e Faz Críticas; (c) Fator 3 explica 13,024\% da variância, correspondendo às categorias Sentimentos Negativos, Receber Crítica e Apresentar Seminários. Destaca-se que o instrumento como um todo apresenta $66,279 \%$ de variância acumulada explicada.

Quanto à Parte 2 do QHC-Universitários, na reaplicação, o índice KMO obtido foi de 0,577 e o teste de esfericidade de Barlett apresentou resultado significativo $(p=0,000)$, os quais indicam adequação dos dados para as análises. De maneira idêntica à validação exploratória apresentada, as categorias comportamentais foram explicadas por dois fatores. O primeiro, denominado Potencialidades, agregou as categorias Situações/assuntos, Comportamento habilidoso, Consequência positiva e Sentimentos positivos. O segundo fator, Dificuldades, agregou Comportamento não habilidoso, Consequência negativa e Sentimentos negativos.

\section{Estudos de Validade Concorrente}

A Tabela 2 apresenta os resultados das análises de comparação entre os grupos clínicos para depressão e ansiedade com os grupos não clínicos.

Conforme a Tabela 2, o QHC-Universitários discriminou dois dos três fatores da Parte 1 do instrumento e os dois fatores da Parte 2, na comparação entre os grupos clínico e não clínico para depressão. Os resultados mostraram que o

Tabela 1. Matriz fatorial, com rotação Varimax, para os resultados de frequência e de classificações comportamentais e contextuais do QHC-Universitários - Partes 1 e 2

\begin{tabular}{|c|c|c|c|}
\hline $\begin{array}{l}\text { Parte 1. Frequências } \\
\text { comportamentais }\end{array}$ & Fator 1 & Fator 2 & Fator 3 \\
\hline Conversação & 0,812 & & \\
\hline Sentimentos positivos & 0,857 & & \\
\hline Sentimentos negativos & & 0,773 & \\
\hline Opiniões & 0,836 & & \\
\hline Fazer críticas & & 0,724 & \\
\hline Receber críticas & & 0,773 & \\
\hline Falar em público & & & 0,777 \\
\hline Apresentar seminários & & & 0,838 \\
\hline $\begin{array}{l}\text { \% da variância explicada pelo } \\
\text { fator }\end{array}$ & 27,278 & 22,178 & 16,823 \\
\hline \% da variância total acumulada & 27,278 & 49,456 & 66,279 \\
\hline $\begin{array}{l}\text { Parte 2. Características } \\
\text { comportamentais }\end{array}$ & \multicolumn{2}{|c|}{ Fator 1} & Fator 2 \\
\hline Situações/assuntos & \multicolumn{2}{|c|}{0,767} & \\
\hline Comportamento habilidoso & \multicolumn{2}{|c|}{0,859} & \\
\hline Comportamento não habilidoso & & & 0,803 \\
\hline Consequência positiva & \multicolumn{2}{|c|}{0,892} & \\
\hline Consequência negativa & \multirow{3}{*}{\multicolumn{2}{|c|}{0,790}} & 0,828 \\
\hline Sentimento positivo & & & \\
\hline Sentimento negativo & & & 0,847 \\
\hline \% da variância explicada pelo fator & \multicolumn{2}{|c|}{39,469} & 32,435 \\
\hline \% da variância total acumulada & \multicolumn{2}{|c|}{39,469} & 71,904 \\
\hline
\end{tabular}


Fator 1 e o Fator 3 da Parte 1 possuem maiores médias para o grupo não clínico para depressão. Na direção esperada, também se verificou que as Potencialidades tiveram maiores médias para o grupo não clínico, enquanto que as Dificuldades tiveram maiores médias para o grupo clínico para depressão. Na reaplicação, não foram encontradas diferenças entre o grupo clínico $(n=12)$ e não clínico para depressão $(n=26)$, tendo o BDI como instrumento critério a partir do teste $t$.

Considerando os achados obtidos pelas Curvas Roc, observa-se bom desempenho do instrumento no que se refere à avaliação dos Fatores de Comunicação (Fator 1 - Parte 1) e Falar em Público (Fator 3 - Parte 1). O mesmo foi verificado para os Fatores 1 e 2 da Parte 2 do QHC-Universitários, correspondentes, respectivamente, a Potencialidades e Dificuldades.

Para Comunicação, a área encontrada sob a curva foi de $0,609$ ( $\mathrm{p}=0,033)$, com erro padrão de 0,050 e intervalo de confiança de $95 \%$ entre 0,511 e 0,707 . O ponto de corte está entre os escores 16,5 e 18,5, indicando que os participantes com tais escores em Frequência de Comunicação estão no escore não clínico e as frequências abaixo desses valores indicaram escore clínico para depressão. Para o Fator 3 - Falar em Público, a área encontrada foi de 0,652 ( $p=$ $0,003)$, com erro padrão de 0,049 e intervalo de confiança de $95 \%$ entre 0,556 e 0,747 . O ponto de corte está no escore 4,5 , indicando que os participantes com esse escore em Frequência de Falar em Público estão no escore não clínico. Abaixo desse valor, sinaliza-se escore clínico para depressão. Para Potencialidades - Fator 1-Parte 2, a área encontrada foi de 0,666 ( $p=0,001)$, com erro padrão de 0,048 e intervalo de confiança de $95 \%$ entre 0,571 e 0,761 . O ponto de corte está entre os escores 66,5 e 72,00. Para Dificuldades - Fator 2
-Parte 2, a área encontrada foi de 0,762 $(p=0,000)$, com erro padrão de 0,042 e intervalo de confiança de $95 \%$ entre 0,679 e 0,844 . O ponto de corte está entre os escores 17,50 e 18,50.

Conforme a Tabela 2, verificou-se que o Fator 3 da Parte 1 do QHC-Universitários discriminou os grupos clínico e não clínico para ansiedade, tendo maiores médias para o grupo não clínico. Os dois fatores da Parte 2 do QHC-Universitários discriminaram os grupos, sendo as Potencialidades mais frequentes para o grupo não clínico e as Dificuldades, para o grupo clínico para ansiedade.

Os resultados obtidos por meio da Curva Roc indicaram que o Fator 3 da Parte 1 do QHC-Universitários e os Fatores 1 e 2 da Parte 2 do QHC-Universitários discriminaram os grupos clínico para ansiedade e não clínico, indicando a adequação do instrumento. Para o Fator 3 - Falar em Público, a área encontrada foi de $0,634(p=0,000)$, com erro padrão de 0,033 e intervalo de confiança de $95 \%$ entre 0,570 e 0,698 . O ponto de corte está no escore 4,5 sinalizando escore clínico para ansiedade. Para Potencialidades - Fator 1 -Parte 2, a área encontrada foi de 0,594 ( $p=0,006)$, com erro padrão de 0,033 e intervalo de confiança de $95 \%$ entre 0,529 e 0,660 . O ponto de corte está entre os escores 78,50 e 71,50. Para Dificuldades - Fator 2 - Parte 2, a área encontrada foi de $0,692(p=0,000)$, com erro padrão de 0,031 e intervalo de confiança de $95 \%$ entre 0,632 e 0,753 . O ponto de corte está entre os escores 16,50 e 17,50 .

Nas comparações entre grupo clínico $(n=11)$ e não clínico para ansiedade $(n=27)$, tendo a Mini-SPIN como critério foram encontradas diferenças entre os grupos: (a) Comunicação e Afeto (clínico = 13,27/7,06 x não clínico $=16,70 / 3,33 ; p=0,047$ ); (b) Enfrentamento (clínico = $4,36 / 2,50$ x não clínico $=5,44 / 3,54 ; p=0,297)$; (c) Falar em Público $($ clínico $=13,27 / 7,06 \times$ não clínico $=16,70 / 3,33 ; p$

Tabela 2. Validade concorrente: médias, desvios padrão e resultados do Teste $t$ Student (2-tailed) na comparação entre os grupos Clínico-Depressão e Clínico-Ansiedade x Não clínico

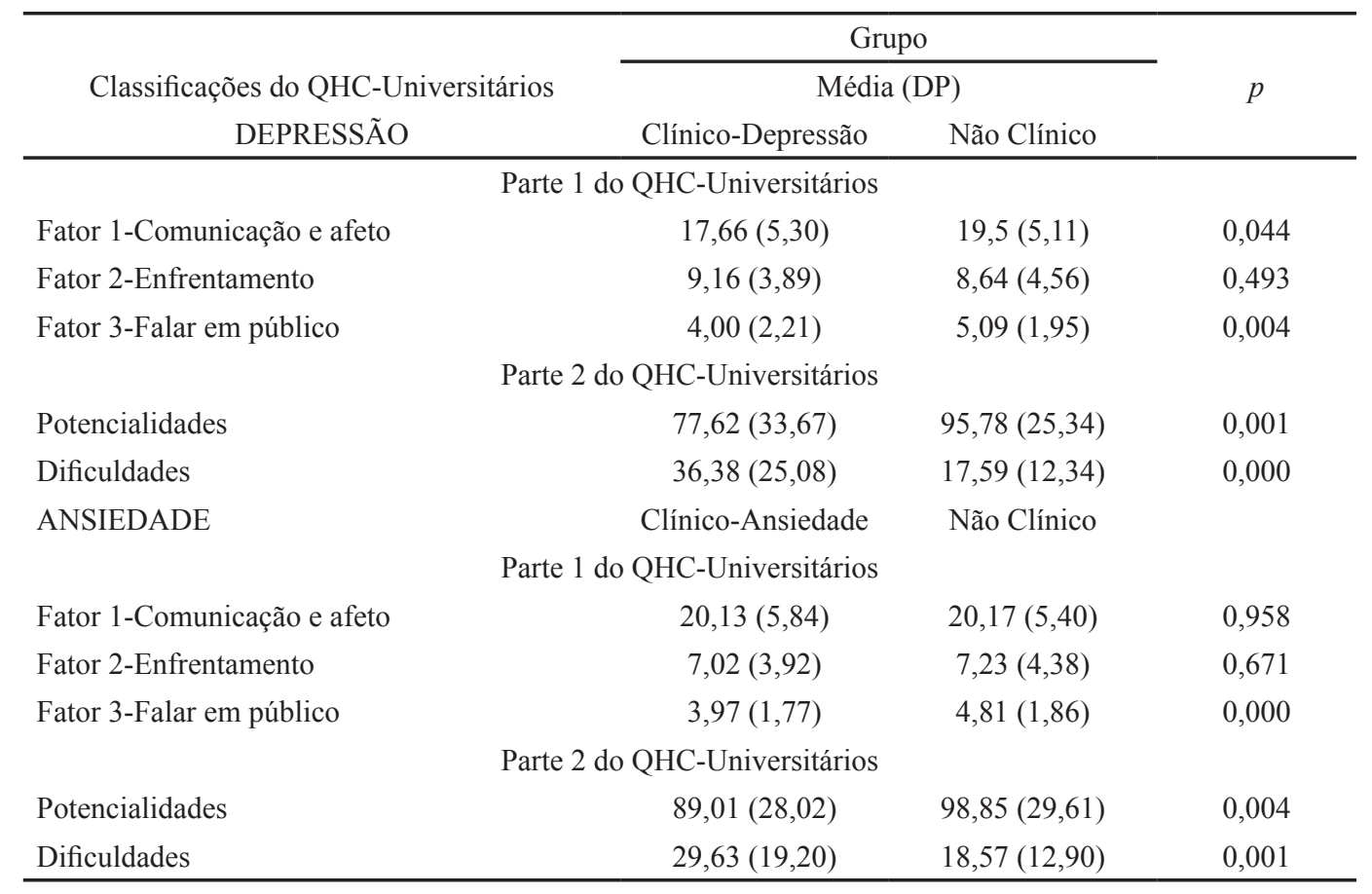


$=0,050) ;(\mathrm{d})$ Potencialidades (clínico $=83,63 / 27,84 \times$ não clínico $=112,44 / 22,31 ; \mathrm{p}=0,008) ;(\mathrm{e})$ Dificuldades (clínico = $41,18 / 17,20$ x não clínico $=15,30 / 2,94 ; p=0,044)$. Quando não houve homogeneidade das variâncias foi considerada a correção de Levene. Como visto, apenas Falar em Público não discriminou os grupos clínico e não clínico para ansiedade, na reaplicação.

Estudo de validade convergente

Conforme a Tabela 3, verificam-se correlações positivas entre o escore total do IHS-Del Prette e as três categorias da Parte 1 do QHC-Universitários: Comunicação e Afeto, Enfrentamento e Fala em Público. Quanto à Parte 2 do QHC-Universitários e os Fatores 2, 3, 4, verificaram-se utilizada na reaplicação, o que sugere a necessidade de novos estudos.

O instrumento, na Parte 2, apresentou dois fatores que discriminaram características positivas (denominadas de Potencialidades) e negativas (denominadas de Dificuldades). Os dois fatores discriminaram os grupos clínicos para depressão e ansiedade, sendo o escore de Potencialidade mais frequente para o grupo não clínico e o de Dificuldade para os grupos clínicos. Tais dados sugerem que QHCUniversitários mostrou-se adequado para mensurar tanto os recursos adaptativos quanto as habilidades sociais como as dificuldades nos relacionamentos interpessoais de universitários, sobretudo aqueles com indicadores

Tabela 3. Validade convergente das categorias do Q-HC-Universitários tendo por referência o IHS-Del Prette

\begin{tabular}{|c|c|c|c|c|c|c|c|}
\hline & Fator 1 & Fator 2 & Fator 3 & Fator 4 & Fator 5 & Outros & IHS_T \\
\hline \multicolumn{8}{|c|}{ Parte 1 do QHC-Universitários } \\
\hline Fator 1-Comunicação/afeto & $0,175^{* *}$ & $0,270 * *$ & $0,139 * *$ & $0,055 * *$ & $0,167 * *$ & $0,152 * *$ & $0,240 * *$ \\
\hline Fator 2-Enfrentamento & $0,117 * *$ & $0,088^{*}$ & --- & $0,060 *$ & $0,046 * *$ & $0,022 *$ & $0,088 *$ \\
\hline Fator 3- Falar em público & $0,334 * *$ & $0,250 * *$ & $0,153 * *$ & $0,379 * *$ & $0,065 * *$ & $0,088 * *$ & $0,333 * *$ \\
\hline \multicolumn{8}{|c|}{ Parte 2 do QHC-Universitários } \\
\hline Fator 1-Potencialidades & $0,294 * *$ & $0,257 * *$ & $0,184 * *$ & --- & $0,204 * *$ & $0,214 * *$ & --- \\
\hline Fator 2- Dificuldades & --- & $-0,095 * *$ & $-0,141 * *$ & $-0,165 * *$ & --- & $-0,112 * *$ & $-0,206^{* *}$ \\
\hline
\end{tabular}

correlações negativas e significativas com o total do IHSDel Prette e com o Fator Dificuldades da Parte 2 do QHCUniversitários. Na reaplicação, considerando o escore total do IHS-Del Prette com os fatores do QHC-Universitários, foram encontradas duas correlações significativas: Falar em Público com correlação positiva de 0,498 e significância de 0,001; Dificuldades com correlação negativa de 0,475 e significância de 0,003 .

\section{Discussão}

O instrumento QHC-Universitários na Parte 1 apresentou três fatores denominados de Comunicação e Afeto, Enfrentamento e Falar em Público, respectivamente. Os Fatores 1 e 3 discriminaram indicadores para depressão e o Fator 3 para ansiedade, os quais correspondem a indicadores de desenvoltura social, cujos déficits são relacionados tanto à depressão quanto à ansiedade, tal como relatado por Edmondson, Conger e Conger (2007). De forma pouco esperada, os Fatores 1 e 2, denominados respectivamente de Comunicação e Afeto e de Enfrentamento, não discriminaram os grupos clínicos para ansiedade, sugerindo que a maioria dos universitários, independente de seus recursos de saúde mental, podem encontrar dificuldades nos comportamentos que compõem esses fatores, como expressar sentimentos negativos, opiniões e lidar com críticas (Bandeira Rocha, Freitas, Del Prette, \& Del Prette., 2006; Bolsoni-Silva, Loureiro, Rosa \& Oliveira2010; Ribeiro \& Bolsoni-Silva, 2011). Na reaplicação do instrumento, apenas parte dos fatores foi mantida e parte das comparações discriminou os grupos, o que pode ter decorrido da amostra reduzida de depressão e ansiedade. Destaca-se, assim, uma das contribuições potenciais do instrumento, pois, do ponto de vista da Psicologia Preventiva, ter um instrumento que avalia fatores de risco e de proteção constitui-se em importante recurso para uma avaliação mais ampla que poderá subsidiar medidas de intervenção eficazes e eficientes (Dozois \& Westra, 2004). Na reaplicação, os resultados foram mantidos.

Os achados também indicaram que a avaliação é mais acurada quando se avalia a frequência e a qualidade com que os comportamentos são emitidos, frente a diferentes situações e interlocutores. Nesse sentido, independente de se considerar a variável saúde mental, a análise das respostas ao instrumento apontou para uma associação entre repertório de habilidades sociais e a resolução de problemas interpessoais, tal como já relatado por diversos estudos (Backer, 2003, Ciarrochi, Deane, \& Anderson, 2002; Cole, Lazarick, \& Howard, 1986; Furtado et. al., 2003). Há de se destacar que tais afirmativas são suposições que precisam de maior comprovação empírica, uma vez que as correlações com o IHS-Del Prette, ainda que significativas, foram moderadas, o que mostra uma interface dos instrumentos no que diz respeito à variável habilidades sociais, mas, por outro lado, aponta para especificidades dos mesmos, especialmente na avaliação diferenciada de potencialidades e dificuldades por parte do QHC-Universitários. Tais dados corroboram e justificam a proposição desse novo instrumento.

$\mathrm{O}$ instrumento, por meio das medidas de potencialidades e dificuldades, tendo por padrão ouro indicadores de saúde mental, permitiu obter notas de corte satisfatórias que favorecem identificar padrões de comportamentos, nos níveis clínicos e não clínico. Tal achado, no que se refere às habilidades sociais, indica outra potencialidade de 
aplicabilidade do instrumento, dado que a amostra avaliada era da comunidade e, nesse sentido, ele poderá ser aplicado para subsidiar programas de prevenção em saúde mental, tal como proposto por Veenman, Wilhelm e Beishuizen (2004). $\mathrm{O}$ uso de instrumentos aferidos com tais características pode auxiliar profissionais da saúde, sobretudo psicólogos, que atuam em centros de apoio a estudantes universitários favorecendo a proposição de medidas preventivas, as avaliações de triagem, o planejamento de medidas de intervenção e a verificação sistemática dos resultados de tais programas.

Considera-se que, ao construir e estabelecer as medidas de fidedignidade e validade do instrumento, os autores ativeramse aos princípios e métodos recomendados pela psicometria, tal como proposto por Pasquali (1999), Pasquali (2000), Streiner (1993), Nunally (1970), Floyd e Widaman (1995). As medidas de fidedignidade, segundo Pasquali (2000), são necessárias para aferir um instrumento, por indicarem o quanto ele é capaz de reproduzir os resultados obtidos. No caso do QHC-Universitários, foram obtidos valores de alfa de 0,657 para as duas partes, que podem ser considerados como aceitáveis (Cortina, 1993). Na aplicação da versão final, com a amostra de 59 universitários, os valores de alfa foram mantidos, reafirmando que as categorias guardaram a devida semelhança na validação do instrumento.

As análises fatoriais auxiliaram a compreensão e a organização das informações colhidas com o instrumento em três categorias para a Parte 1 (Comunicação e Afeto, Enfrentamento e Falar em Público) e a Parte 2 em Potencialidades e Dificuldades, explicando 66,279\% e $71,904 \%$ de variância, respectivamente. Os resultados obtidos permitiram afirmar que o instrumento tem características multifatoriais, pois possui três fatores para a Parte 1 e dois fatores para a Parte 2 e, também, permitiu caracterizar as tarefas comportamentais, uma vez que descreveu as respostas dos universitários frente a diferentes contextos e as consequências que obtêm, tendo por fonte de informação os seus relatos verbais. Essa modalidade de avaliação pode contribuir com programas de intervenção específicos, o que vai ao encontro do proposto por Gerk e Cunha (2006) quanto à preocupação da universidade de avaliar e intervir junto a essa população, de forma a evitar problemas de saúde mental e abandono escolar.

No caso do estudo conduzido com o QHC-Universitários, foram aferidas as validades de construto e de critério concorrente e convergente, sendo o IHS-Del Prette (Del Prette \& Del Prette, 2001) tomado como uma medida de construto semelhante (habilidades sociais) e a MiniSpin, o BDI e a SCID-IV como medidas clínicas (saúde mental). Os resultados da validade convergente tendo o IHS-Del Prette como medida de referência demonstraram que o QHC-Universitários avalia o construto habilidades sociais, pois suas categorias positivas tiveram correlação com fatores e escore total do IHS-Del Prette (Comunicação e Afeto, Enfrentamento e Fala em Público $\neg-$ Parte 1 e Potencialidades - Parte 2). No entanto, as medidas de Dificuldade correlacionaram-se negativamente com os fatores e o Escore total do IHS-Del Prette, o que foi mantido nos resultados da reaplicação. Esse dado sugere que o QHC-Universitários avalia não só as habilidades sociais, mas também as dificuldades interpessoais, as quais são reconhecidas como fatores de risco para a adaptação (Dozois $\&$ Westra, 2004). Pode-se afirmar que o construto habilidades sociais foi, de fato, mensurado pelo QHC-Universitários, pois foram encontradas correlações significativas com o IHS-Del Prette (Del Prette \& Del Prette, 2001), instrumento aferido para a população de universitários brasileiros.

Pasquali (2000) também recomenda que, para maximizar a validação, é interessante utilizar várias medidas. No caso do presente estudo, análises discriminativas foram utilizadas para reduzir os itens do QHC-Universitários, tendo como critério medidas de saúde mental (indicadores de depressão, fobia social e transtorno psiquiátrico). As comparações entre os grupos clínico e não clínico permitiram as análises de validação concorrente, tendo o diagnóstico como variável de critério.

Com base nos estudos psicométricos conduzidos e nos resultados identificados, o QHC-Universitários parece ter peculiaridades que o distinguem de outros instrumentos similares propostos na literatura nacional, o que, aliado às suas qualidades psicométricas, justificam a sua proposição e utilidade. O QHC-Universitários configura-se como um avanço em relação à Escala de Avaliação de Vida Acadêmica (Vendramini et al., 2004) no que se refere a identificar comportamentos relacionados com a saúde mental do universitário, bem como na ampliação das medidas de relacionamento, pois o QHC-Universitários, conforme mencionado, avalia os efeitos das respostas emitidas, além das respostas e das condições nas quais ocorrem. Por outro lado, o QHC-Universitários avalia um número menor de dimensões em comparação com a Escala de Avaliação de Vida Acadêmica (ambiente universitário, compromisso com o curso, habilidade interpessoal do estudante, envolvimento em atividades não obrigatórias e condições para o estudo e desempenho acadêmico).

No que se refere ao Inventário de Assertividade Rathus (Ayres, 1994) e ao Inventário de Habilidades Sociais - IHS (Del Prette \& Del Prette, 2001), considera-se que o QHCUniversitários, acrescenta informações sobre habilidades sociais na universidade no que se refere a (a) avaliar habilidades sociais contingentes a contextos diversos e a diferentes interlocutores, identificadas como problemáticas pelos avaliados, como proposto por Ribeiro e Bolsoni-Silva (2011); (b) permitir obter relação entre categorias avaliadas e indicadores de saúde mental, sobretudo fobia social e depressão, transtornos prevalentes na população geral; (c) avaliar contextos, respostas e consequências de uma ampla gama de condições abordadas como temática avaliada pelo instrumento, o que está de acordo com os pressupostos de estudos de prevenção (Dozois \& Westra, 2004), que avaliam, para além de dificuldades, também potencialidades; (d) avaliar também respostas consideradas como não habilidosas e suas consequências diante de demandas interpessoais, as quais foram correlacionadas negativamente com o IHS-Del Prette, colocando em foco as potencialidades do QHC-Universitários para nortear programas de prevenção e intervenção, tal como sugerido para a prevenção de transtornos mentais (World Health Organization, 2004). 


\section{Considerações Finais}

Verificou-se que o QHC-Universitários atendeu plenamente aos critérios psicométricos quanto aos estudos de fidedignidade, validade de construto e validade concorrente, e parcialmente quanto à validade convergente. Destaca-se uma limitação quanto à generalização dos resultados, visto que o estudo foi conduzido a partir de uma amostra identificada em única universidade.

Considera-se como diferencial que o instrumento avalia tanto frequência quanto características dos comportamentos, favorecendo mapear se os problemas estão relacionados à frequência ou à qualidade das interações, permitindo, além da identificação das habilidades sociais, a caracterização dos contextos de dificuldades, por meio da descrição das contingências de respostas frente a diferentes interlocutores. Considerando as boas qualidades psicométricas, destaca-se que o QHC-Universitários poderá ser utilizado tanto no contexto aplicado como de novas pesquisas. Recomendase que o instrumento seja utilizado em clínicas escola, em serviços de apoio e orientação acadêmica a universitários e em ambulatórios de saúde mental, com a função de nortear a proposição de programas preventivos e sistematizar os procedimentos de triagem. Adicionalmente, por permitir avaliar diferencialmente repertórios preditivos de transtorno de ansiedade e de depressão, o seu uso pode favorecer a verificação da efetividade de intervenções. Tais possibilidades de aplicação poderão ser fonte de novos estudos.

\section{Referências}

Adlaf, E. M., Gliksman L., Demers, A., \& Newton-Taylor, B. (2001). The prevalence of elevated psychological distress among Canadian undergraduates: Findings from the 1998 Canadian Campus Survey. Journal of American College Health, 5(2), 67-72.

Ayres, L. S. M. (1994). Uma escala brasileira para a medida da assertividade. (Unpublished master's thesis). Universidade Gama Filho, Rio de Janeiro.

Backer, S. R. (2003). A prospective longitudinal investigation of social problem- solving appraisals on adjustment to university, stress, health and academic motivation and performance. Personality and Individual Differences, 35(3), 569-591.

Bandeira, M., Costa, M. N, Del Prette, Z. A. P., \& Del Prette, A. (2000). Qualidades psicométricas do Inventário de Habilidades Sociais (IHS): Estudo sobre a estabilidade temporal e a validade concomitante. Estudos de Psicologia, 5(2), 401-419.

Bandeira, M., Rocha, S. S., Freitas, L. C., Del Prette, Z. A. P., \& Del Prette, A. (2006). Habilidades sociais e variáveis sociodemográficas em estudantes do ensino fundamental. Psicologia em Estudo, 11(3), 541-549.

Barreto, M. C. M., Pierri, M. R. S. R., Del Prette, Z. A. P., \& Del Prette, A. (2004). Habilidades sociais entre jovens universitários: Um estudo comparativo. Revista Matemática Estatística, 22(1), 31-42.

Bolsoni-Silva, A. T., Loureiro, S. R., Rosa, C. F., \& Oliveira, M. C. F. A. (2010). Caracterização das habilidades sociais de universitários. Contextos Clínicos, 3(1), 62-75.
Bolsoni-Silva, A. T. (2011). Habilidades sociais e saúde mental de estudantes universitários: Construção e validação do $Q-A C C$ $V U$ e estudos clínicos em análise do comportamento. Tese de Livre-Docência, Faculdade de Ciências, Universidade Estadual Paulista Júlio de Mesquita Filho, Bauru, SP.

Cercheari, E.A.N.; Caetano, D. \& Facenda,O. (2005). Prevalência de transtornos mentais menores entre estudantes universitários. Estudos de Psicologia, 10(3), 413-420.

Ciarrochi, J., Deane, F. P., \& Anderson, S. (2002). Emotional intelligence moderates the relationship between stress and mental health. Personality and Individual Differences, 32(2), 197-209.

Cole, D. A., Lazarick, D. L., \& Howard, G. S. (1986). Construct validity and the relation between depression and social skill. Journal of Counseling Psychology, 34(3), 315-321.

Cortina, J. M. (1993). What is coefficient Alfa? An examination of theory and applications. Journal of Applied Psychology, 78(1), 98-104.

Connor, K. M., Kobak, K. A., Churchill, L. E., Katzelnick, D., \& Davidson, J. R. (2001). Mini-Spin: A brief screening assessment for generalized social anxiety disorder. Depression and Anxiety, 14, 137-140.

Cunha, J. A. (2001). Manual da versão em português das Escalas Beck. São Paulo: Casa do Psicólogo.

Del-Ben, C. M., Vilela, J. A. A., Crippa, J. A. S., Hallak, J. E. C., \& Zuardi, A. W (2001). Confiabilidade teste-reteste da Entrevista Clínica Estruturada para o DSM-IV (SCID) versão clínica. Revista Brasileira de Psiquiatria, 23(6), 156-159.

Del Prette, A., Del Prette, Z. A. P., \& Barreto, M. C. M. (1998). Análise de um inventário de habilidades sociais (IHS) em uma amostra de universitários. Revista Psicologia: Teoria e Pesquisa, 14(3), 219-228.

Del Prette, A., \& Del Prette, Z. A. P. (1983). Análise do repertório assertivo em estudantes de Psicologia. Revista de Psicologia, 1, 15-24.

Del Prette, A., \& Del Prette, Z. A. P. (2001). Inventário de habilidades sociais (IHS- Del Prette): Manual de aplicação, apuração e interpretação. São Paulo: Casa do Psicólogo.

Dozois, D. J. A, \& Westra, H. A. (2004). The nature of anxiety and depression: Implications for Prevention. Em D. J. A Dozois \& K. S. Dobson (Orgs.), The Prevention of Anxiety and Depression. Theory, research, and practice (pp. 9-41). Washington: American Psychological Association.

Edmondson, C. B., Conger, J. C., \& Conger, A. J. (2007). Social skills in college students with high trait anger. Journal of Social and Clinical Psychology, 26(5), 575-594.

Facundes, V. L. D., \& Ludermir, A. B. (2005). Common mental disorders among health care students. Revista Brasileira de Psiquiatria, 27(3), 194-200.

Fang, D., Young, C., Golshan, S., Fellows, I., Moutier, C., \& Zisook, S. (2010). Depression in premedical undergraduates: A cross-sectional survey prim care companion. The Journal of Clinical Psychiatry, 12(6). PCC.10m00958. Recuperado em 20 de março de 2013, de http://www.ncbi.nlm.nih.gov/pmc/ articles/PMC3067995/.

Finger, I. R. (2008). Validade de construto do Inventário de depressão de Beck-II (BDI-II) em uma população universitária. (Unpublished master's thesis). Programa de Pós Graduação da Faculdade de Psicologia da Pontifícia Universidade Católica do Rio Grande do Sul, Porto Alegre, RS. 
Floyd, F. J., \& Widaman, K. F. (1995). Factor analysis in the development and refinement of clinical assessment instruments. Psychological Assessment, 7(3), 286-299.

Furtado, E. S., Falcone, E. M. O., \& Clark, C. (2003). Avaliação do estresse e das habilidades sociais na experiência acadêmica de estudantes de medicina de uma universidade do Rio de Janeiro. Interação em Psicologia, 7(2), 43-51.

Gerk, E., \& Cunha, S. M. (2006). As habilidades sociais na adaptação de estudantes ao ensino superior. Em M. Bandeira, A. Del Prette \& Z. Del Prette (Orgs.), Estudos sobre habilidades sociais e relacionamento interpessoal (pp. 181-198). São Paulo: Casa do Psicólogo.

Goldiamond, I. (2002). Toward a constructional approach to social problems: Ethical and constitucional issues raised by Applied Behavior Analysis. Behavior and Social Issues, 11, 108-197. Original publicado em 1974

Mcgaha, V., \& Fitzpatrick, J. (2005). Personal and social contributors to dropout risk for undergraduate students. College Student Journal, 39(2), 287-297.

Neves, M. C. C., \& Dalgalarrondo, P. (2007). Transtornos mentais auto-referidos em estudantes universitários. Jornal Brasileiro de Psiquiatria, 56(4), 237-244.

Nunnally, J. C. (1970). Introduction to psychological measurement. New York: McGraw-Hill.

Osório, F. (2008). Transtorno de ansiedade social: Validação de instrumentos de avaliação (Unpublished doctoral dissertation). Programa de Pós Graduação em Saúde Mental da Universidade de São Paulo, Ribeirão Preto, SP.

Osório, F. L., Crippa, J. A. S., \& Loureiro, S. R. (2007). A study of the discriminative validity of a screening tool (Mini-Spin) for social anxiety disorders applied to Brazilian university students. European Psychiatry (Paris), 22(4), 1-5.

Pasquali, L. (1999). Testes referentes a construto: Teoria e modelo de construção. Em L. Pasquali (Ed.). Instrumentos psicológicos: Manual prático de elaboração (pp. 37-71). Brasília: LabPAM/ IBAPP.
Pasquali, L. (2000). Princípios de elaboração de escalas psicológicas. Em C. Gorenstein, L. H. S. G. Andrade \& A. W. Zuardi (Eds.), Escalas de avaliação clínica em Psiquiatria e Psicofarmacologia (pp. 15-21). São Paulo: Lemos.

Ramírez, M. T. G., Hernández, R. L., \& García-Campayo, J. (2009). Relación entre la depresión, la ansiedad y los sintomas psicosomáticos en uma muestra de estudiantes universitarios del norte de México. Revista Panamericana Salud Publica, 25(2), 141-145.

Ribeiro, D., \& Bolsoni-Silva, A. T. (2011). Potencialidades e dificuldades interpessoais de universitários: Estudo de caracterização. Revista Acta Comportamentalia, 19(2), 205224.

Roberts, L. W., Wamer, T. D., Lyketsos, C., Frank, E., Ganzini, L., \& Carter, D. (2001). Perceptions of academic vulnerability associated with personal illness: A study of 1,027 students at nine medical schools. Comprehensive Psychiatry, 42(1), 1-15.

Streiner, D. L. (1993). A checklist for evaluating the usefulness of rating scales. Canadian Journal of Psychiatry, 78(2), 140-148.

Sturmey, P. (1996). Functional analysis in clinical psychology. England: John Wiley \& Sons.

Veenman, M. V. J., Wilhelm, P., \& Beishuizen, J. J. (2004). The relation between intellectual and metacognitive skills from a developmental perspective. Learning and Instrucion, 14(1), 89-109.

Vendramini, C. M. M., Santos, A. A. A., Polydoro, S. A. J., Sbardelini, E. T. B., \& Serpa, M. N. F. (2004). Construção e validação de uma escala sobre avaliação da vida acadêmica (EAVA). Estudos de Psicologia, 9(2), 259-268.

Woods, P., Reed, V., \& Collins, M. (2003). Exploring core relationships between insight and communication and social skills in mentally disordered offenders. Journal of Psychiatric and Mental Health Nursing, 10, 518-525.

World Health Organization. (2004). Prevention of mental disorders - effective interventions and policy options. Paris: França. Recuperado em 20 de março de 2013, de: http://www.who.int/ mental_health/evidence/en/prevention_of_mental_disorders sr.pdf. 